\title{
METASTATIC TUBERCULOUS ABSCESSES
}

BY

\author{
F. J. W. MILLER and MAUREEN CASHMAN \\ From the Department of Child Health, University of Durham
}

(RECEIVED FOR PUBLICATION AUguST 22, 1954)

In the past three years we have studied several children suffering from chronic tuberculous ulceration of the skin. These ulcers, usually multiple, were formed by the rupture through the skin of a tuberculous abscess which began beneath it. The ulcer slowly healed leaving a depressed scar.

There are brief references to this type of ulceration in both paediatric and dermatological textbooks, but we cannot find a clinical description of the lesion clearly differentiating it from an abscess arising from tuberculous lymphadenitis or from a focus in bone, or from tuberculosis cutis; nor can we find any reference to the relation of the lesions to the time of primary infection.

We think, therefore, that it will be useful to describe the course of these uncommon lesions and demonstrate their relation to the primary infection.

Four cases are recorded. We have seen three others, but our notes are insufficient to include them. The seven cases occurred in approximately 1,000 children suffering from tuberculosis seen in the hospitals and various clinics in the city. The incidence, therefore, cannot be more than $1 \%$ of tuberculous children and probably is much less.

\section{Case Reports}

Case 1. M.R., a girl of 8 years, one of a family of six children living on Tyneside, was brought to hospital in July, 1951. About nine months earlier her mother found a small painless lump under the child's left buttock. During the next six months the lump turned blue slowly and eventually discharged blood-stained fluid. Three months afterwards a similar lump was seen on the right thigh, and within the next two months two others, one on the left buttock and one in the right popliteal fossa. This last lesion discharged following a kick.

The lumps were never painful. They were first under the skin and separate from it, but later became attached as they broke slowly through to discharge. Her general health remained good and she was sent to hospital because the resulting ulcers were not healing.

One of her elder sisters had been in a sanatorium three years earlier suffering from a tuberculous pleural effusion and a lung lesion, and there were two other young adults with pulmonary tuberculosis living near.
On examination, she was a sturdy child with two open ulcers, one on the right buttock and one behind the left knee; the scar of one other on the left buttock and a small unruptured abscess above this (Fig. 1). The ulcers were roughly circular with narrow, blue-red margins fading into the skin; the edges were undermined and the floors were of reddish granulations covered with thick yellow pus. The abscess which had not opened was painless, hard, fixed to the skin but free over the deeper tissues. The overlying skin was bluish-red with some superficial desquamation.

The tuberculin skin reaction was positive; the Wasser-

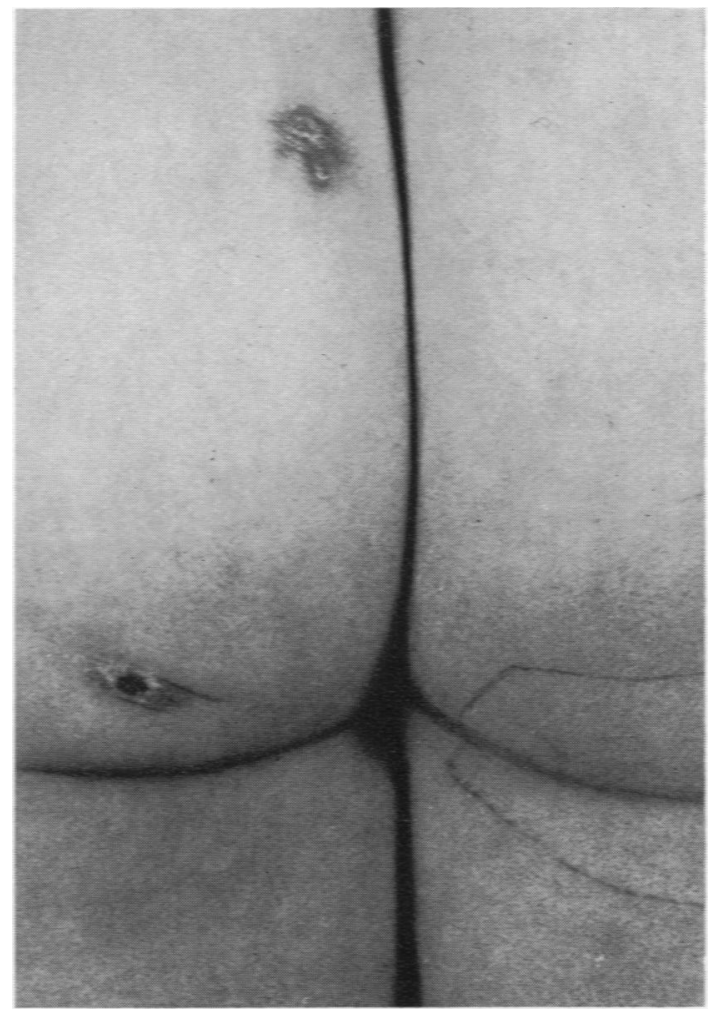

Fig. 1.-Case 1 : left buttock, showing an ukcer scar and an unruptured abscess above. 
mann and Kahn tests were negative. A radiograph of the lungs showed a small right pleural effusion with a segmental opacity, of the type commonly seen in primary tuberculosis, in the upper lobe. Two of the ulcers were examined histologically. The reports (Dr. J. Hart Mercer) stated:

-Tubercle follicles are demonstrable at the margins of the otherwise non-specific granulation tissue lining the base of the dermal uker ... This ulcer, too, shows unmistakable tuberculosis.

She received no specific treatment but was transferred to a children's sanatorium. There, the local lesions healed in a few weeks; the radiological shadows in the chest cleared more slowly. In April, 1952, she went home and has remained well since: no further skin lesions have developed; recent radiographs show faint cakification in the right paratracheal glands.

This child developed multiple abscesses in the early post-primary stage, probably within 12 months of infection.

Case 2. C.W., a girl, aged 12 years, was admitted to hospital in November, 1949, with fever, abdominal pain and tenderness. Her tuberculin reaction was strongly positive and a radiograph of the chest was normal. The pain settled in a few days and she was allowed home with a provisional diagnosis of primary abdominal tuberculosis. Remaining well for four months, she was then readmitted in a further attack of abdominal pain and found to have a left-sided pleural effusion. She was

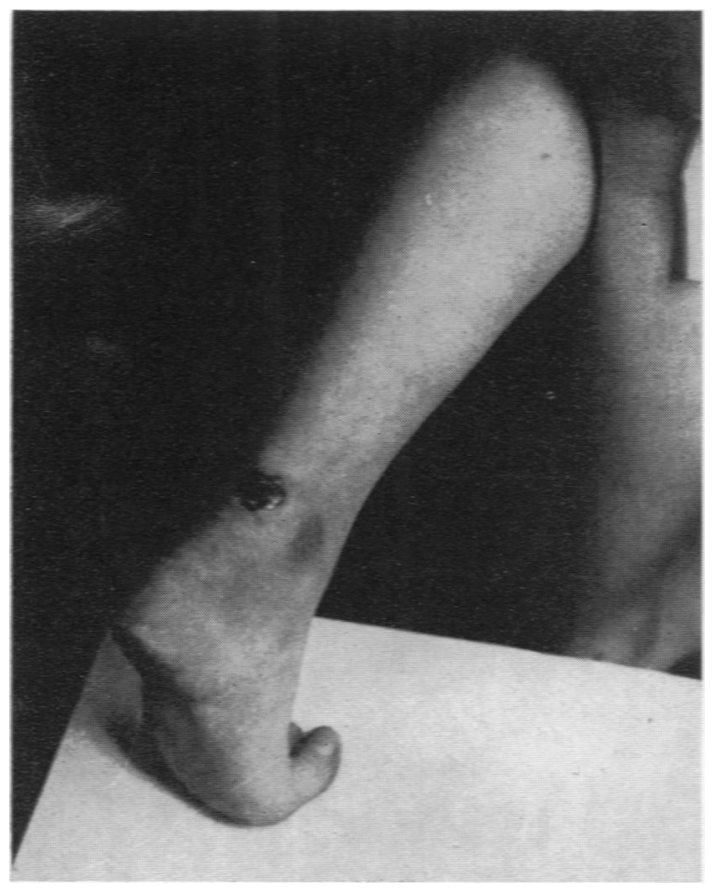

Fig. 2.-Case 2: the ukcer on the back of the left ankle. transferred to a children's sanatorium where she stayed until March, 1951, 16 months after the initial illness. During this time she had right-sided chest pain followed by a pleural effusion. This cleared without complication and on discharge she was well.

Some 14 months later, and two and a half years after the primary infection, while staying in another part of the country, she developed a tender, fluctuant swelling over the upper part of the right tibia and was sent to an orthopaedic hospital. No details of her treatment are available but she had a plaster splint applied and returned to her home in Newcastle a few days later, where she was again seen at hospital. Radiographs of the knee showed no bony abnormality, and she was watched as an outpatient for three months. During this time she developed an uker over the left tendo achillis, and on questioning admitted that a lump had been there for some four months (Fig. 2). The abscess at the upper end of the right tibia had remained and she was also found to have a discharging 'sore' over the metatarso-phalangeal joint of the second left toe.

The Wassermann reaction was negative. Radiographs of the knee and ankle again showed no bony abnormality. Pus aspirated from the abscess over the right tibia was sterile.

She was then readmitted to hospital, the abscess on the tibia was excised, and a section taken from the uker on the left ankle. The pathological report (Dr. R. PatersonSmith) stated that histological examination of both tissues showed fibro-caseous tuberculosis, the former a cyst filled with caseous material and the latter an uker with tuberculous granulation tissue and caseation.

Following three month' treatment with streptomycin and P.A.S. the operation site and the ukers were all soundly healed. Radiographs were still normal and she was discharged. She is now working away from Newcastle but is reported to be well.

In this case the abscesses started at least two and a half years after the primary infection and all three lesions appeared within six months.

Case 3. N.L., a girl, aged $7 \frac{1}{9}$ years, was first seen in 1950 complaining of abdominal pain. The tuberculin reaction was probably positive at that time but the records are not completely clear on this point.

She returned two and a half years later having been well until three months previously when she had a severe attack of scarlet fever. Within two weeks of her discharge from the fever hospital she noticed a small lump in the front of the right thigh just below the groin. This turned red and after about two more weeks discharged blood-stained pus. A similar but larger lump then developed on the outer side of the left knee and became fluctuant. Neither lesion was painful. She had been given penicillin by her family doctor, and when this had no effect was referred to hospital (Figs. 3 and 4).

Her tuberculin reaction was positive; sterile pus was aspirated from the lump at the knee. Following aspiration the abscess leaked and tubercle bacilli were cultured from the pus. Biopsy of the skin showed active tuberculosis. Radiographs of the chest and abdomen 


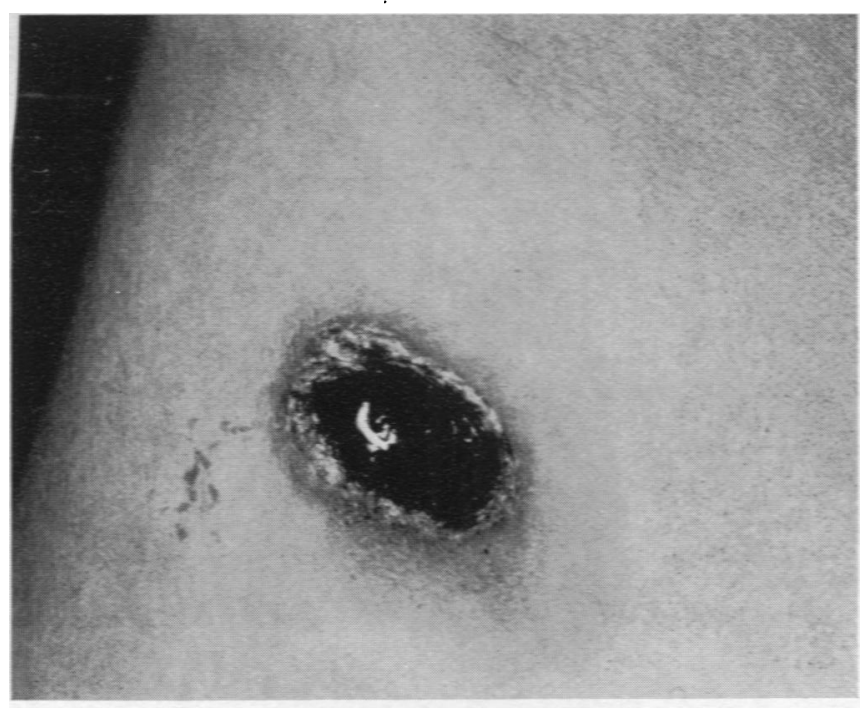

Fwg. 3.-Case 3: the uker on the right thigh.

$\frac{3}{3}$

Fig. 4.-Case 3: the ulcer over the head of the left fibula.

Fig. 5. Case 3: the right thigh ulcer healed three months later.

Fig. 6-Case 3: the ulcer at the left knee, showing breakdown and formation of the secondary ubcer.

FiG. 3.

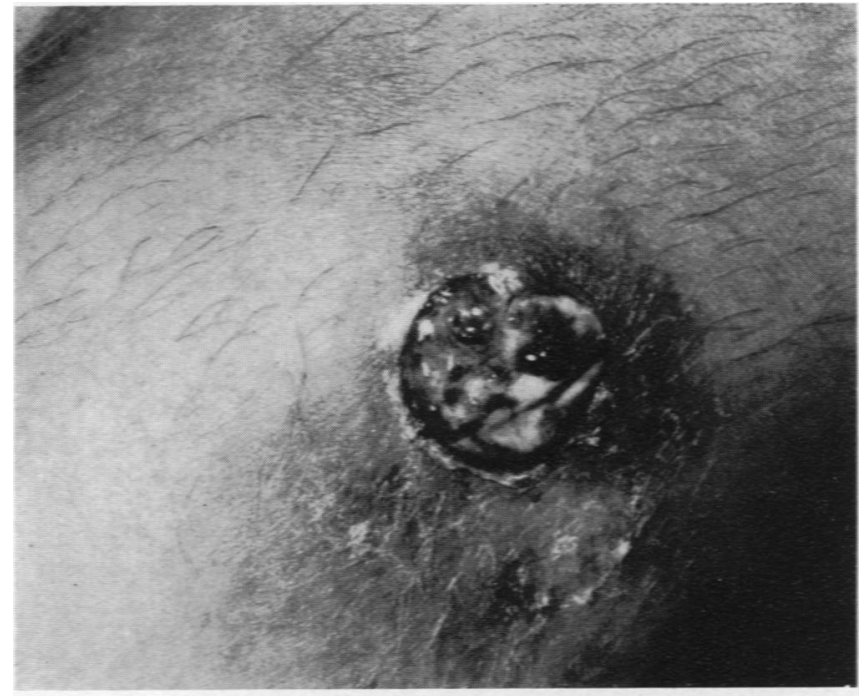

FIG. 4.
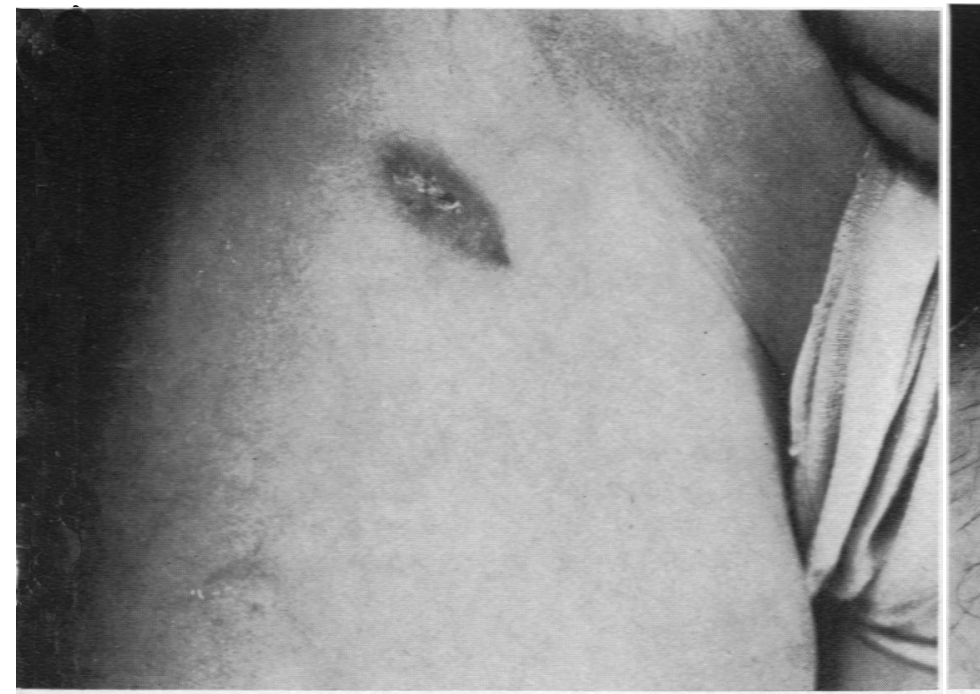
showed no calcification but there was a tuberculous focus in the transverse process of the right eleventh rib. There was no evidence of bone disease in the right femur or left tibia or fibula.

She was given a short course of streptomycin. The thigh lesion healed after scabbing over (Fig. 5). The skin over the abscess at the knee broke down, forming two ukers separated by a bridge of unhealthy skin. For two or three weeks the base remained covered with a dirty slough, but this cleared slowly, leaving a granulating base (Fig. 6). This uker took several months to heal, but a year later there was a firm depressed scar, the rib lesion had disappeared, and the girl was well (Fig. 7).

The lesion in this case became manifest about two years after the primary infection following a streptococcal sore throat. We do not know if the spread occurred then or if, at a time of reduced resistance, the abscesses developed from bacilli previously deposited.

Case 4. M.N., a boy, aged 10 years, was seen in February, 1954, with a fluctuant swelling at the lateral border of the right pectoralis major. Twelve days previously he had fallen off a sledge and the next day noticed a lump. Probably the fall had only drawn his attention to it for there was no bruising; the lump was painless and fluctuant, about 2 in. in length. There was no gland swelling nor any skin lesion (Fig. 8).

The boy was not ill and we could not find any clinical or radiological evidence of primary infection or under-

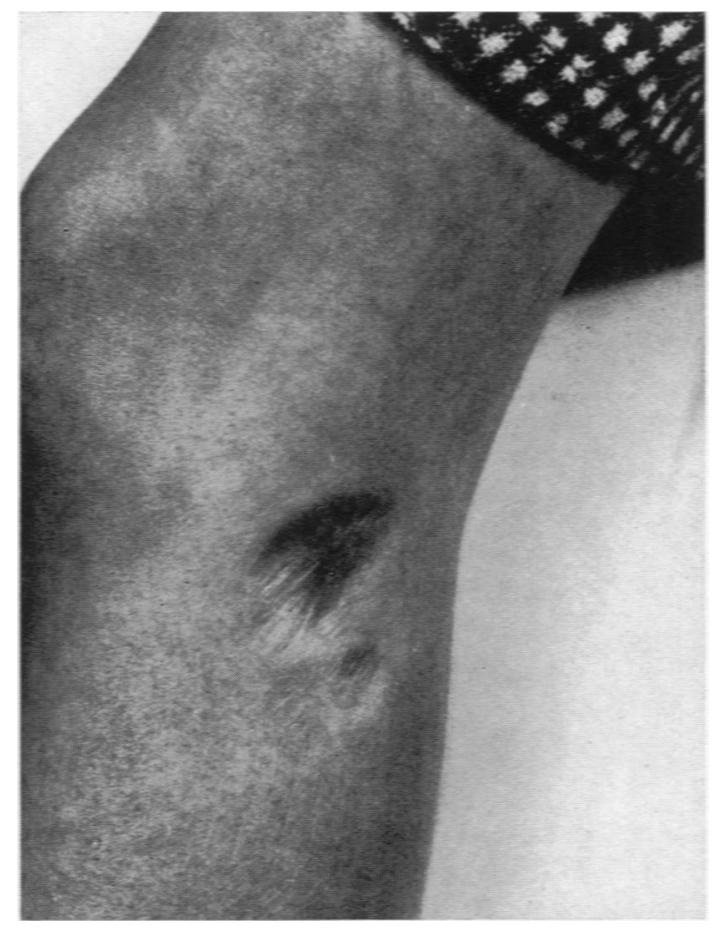

Fig. 7.-Case 3: the scar over the head of the fibula 12 months later. lying bone infection, though his tuberculin reaction was positive. Pus aspirated from the abscess grew tubercle bacilli. During the next few days the lump increased in

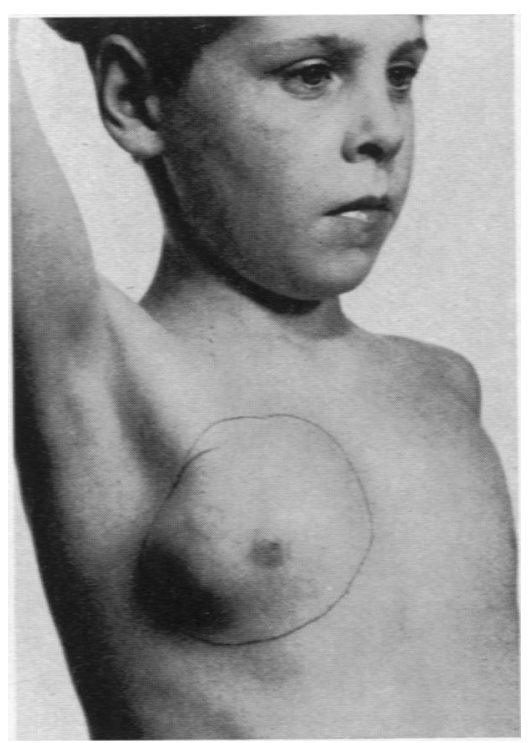

Fig. 8.-Case 4: the abscess presenting at the lateral border of the pectoralis major.

size, spreading under the pectoralis major so that pressure on the muscle produced a distinct bulge. The skin was becoming discoloured and therefore the abscess was drained. With streptomycin and isoniazid therapy the wound healed by first intention. The boy has remained well.

This boy had a single deep abscess and as yet has no clinical or radiological signs to locate the site of the primary lesion. This suggests that the infection is recent and we may later find calcification appearing in the neck, chest or abdomen.

\section{Discussion}

In each of these cases the history is of an abscess or abscesses forming in the subcutaneous tissue and slowly involving the overlying skin. All except the last have discharged blood-stained pus and left indolent ulcers which have taken some weeks or months to heal. With one exception (not seen by us at that stage) these abscesses were painless. They were unrelated to trauma or skin wound. In two, and probably in a third, primary tuberculous infection was already known to have taken place, and the fourth was proven to be tuberculous although the site and time of the primary infection are not yet known.

All these facts can be fitted to the natural history of primary tuberculosis by assuming that in each 
case the abscess had its origin in haematogenous spread from a primary infection; in Cases 1 and 4 during the first year after infection and in Cases 2 and 3 in the third year afterwards.

None of the children had clinical miliary tuberculosis, although in three of the four cases there were multiple foci present - either before the abscesses appeared or at the same time. Two children had a pleural effusion and one a tuberculous rib. In none of the cases was there enlargement or caseation of the regional lymphatic glands draining the site of the abscess or involvement of underlying bone.

These cases are clinically similar to tuberculous abscesses occurring after intramuscular or subcutaneous injections, whether they arise from the deposition of bacilli during the stage of bacillaemia at the site of tissue damage or are introduced by the injection, providing in each case that the subject has already undergone primary infection (Ebrill and Elek, 1946; Hounslow, 1946; Forbes and St. Clair Strange, 1949). An identical abscess has been seen after auto-inoculation of living tubercle bacilli (Chien and Wiggins, 1954). In tuberculin-negative persons injection of tubercle bacilli causes a primary tuberculous abscess with involvement and caseation of the regional glands.
The common types of tuberculous abscess resemble those described above, but are easily distinguishable. Caseating glands from a primary complex may form a cold abscess, but the underlying gland mass will always be found if sought. The origin of a tuberculous abscess arising from an infected bone or joint can be demonstrated by radiography of the affected part.

\section{Summary}

Four cases of metastatic tuberculous abscess in children are described, and their place in the natural history of tuberculosis discussed. They are presumed to be haematogenous spread from a primary infection occurring during a period of bacillaemia or of lowered resistance.

We would like to thank Dr. G. A. Neligan for referring Case 1 and Mr. Michael James who allowed us to see Case 2 when she was admitted to hospital; Dr. C. A. Green for the bacteriological reports; and Mr. J. D. Duncan, Director of the Department of Photography, for the photographs.

REFERENCES

Chien, J. T. T. and Wiggins, M. L. (1954). Amer. Rev. Tuberc., $69,818$.

Ebrill, D. and Elek, S. D. (1946). Lancet, 2, 379.

Forbes, G. B. and Strange, F. G. St. Clair (1949). Ibid., 1, 478. Hounslow, A. G. (1946). Ibid., 2, 617. 